\section{European \\ Surgical Research}

Eur Surg Res 2020;61:163-176

\title{
Complement Component 3 Is Required for Tissue Damage, Neutrophil Infiltration, and Ensuring NET Formation in Acute Pancreatitis
}

\author{
Johan Linders $^{a} \quad$ Raed Madhi $^{a} \quad$ Matthias Mörgelin $^{b} \quad$ Ben C. King ${ }^{c}$ \\ Anna M. Blom ${ }^{c}$ Milladur Rahman ${ }^{a}$ \\ a Department of Clinical Sciences, Section for Surgery, Lund University, Malmö, Sweden; \\ ${ }^{b}$ Colzyx, Medicon Village, Lund, Sweden; ' Section of Medical Protein Chemistry, \\ Department of Translational Medicine, Lund University, Malmö, Sweden
}

\author{
Keywords \\ Complement · Chemokines · Inflammation · Leukocyte $\cdot$ Pancreas
}

\begin{abstract}
Background: Neutrophil extracellular traps (NETs) are known to play an important role in the pathophysiology of acute pancreatitis (AP). Activation of the complement cascade has been shown to occur in AP. The aim of this study was to examine whether complement component 3 is involved in the generation of NETs in AP. Methods: AP was induced in wild-type and C3deficient mice by retrograde infusion of taurocholate into the pancreatic duct. Blood, lung, and pancreas tissue were collected and MPO activity was determined in lung and pancreas tissue. Histological examination of the inflamed pancreas was performed. Plasma levels of CXCL2, MMP-9, IL-6, and DNA-histone complexes as well as pancreatic levels of CXCL1 and CXCL2 were determined by use of enzyme-linked immunosorbent assay. NETs were detected in the pancreas by electron microscopy. The amount of MPO and citrullinated histone 3 in neutrophils isolated from bone marrow was examined using flow cytometry. Results: In C3deficient mice, challenge with taurocholate yielded much fewer NETs in the pancreatic tissue compared with wild-type controls. Taurocholate-induced blood levels of amylase, tissue injury, and neutrophil recruitment in the pancreas were markedly reduced in the mice lacking C3. Furthermore, MPO levels in the lung, and plasma levels of IL-6, MMP-9, and CXCL2 were
\end{abstract}

J.L. and R.M. contributed equally to this work.

\section{Karger ${ }^{\prime \prime}$}


significantly lower in the C3-deficient mice compared to wild-type mice after the induction of AP. In vitro studies revealed that neutrophils from C3-deficient mice had normal NET-forming ability and recombinant C3a was not capable of directly inducing NETs formation in the wildtype neutrophils. Conclusion: C3 plays an important role in the pathophysiology of AP as it is necessary for the recruitment of neutrophils into the pancreas and ensuring NETs formation. Targeting C3 could hence be a potential strategy to ameliorate local damage as well as remote organ dysfunction in AP.

(C) 2021 The Author(s)

Published by S. Karger AG, Basel

\section{Introduction}

Severe acute pancreatitis (AP) poses a major challenge to clinicians due to the lack of specific treatments [1]. AP is still associated with a high risk of morbidity and mortality due to lack of complete understanding of the basic pathophysiology [2]. Activation of trypsinogen to trypsin constitutes the starting point of the pathogenesis of severe AP [3]. In the early phase of severe AP, proteolytic activation plays a significant role by promoting the recruitment of neutrophils into the inflamed organ. It is well documented that trypsin activation, neutrophil recruitment, and microvascular perfusion failure are key components in the development of severe AP [4, 3]. CXC chemokines, such as CXCL1 and CXCL2, are known to activate and attract neutrophils to the sites of inflammation $[5,6]$. Numerous studies have shown that neutrophil recruitment is a rate-limiting step in the activation of trypsinogen [7] and development of severe AP $[3,8]$. Activated neutrophils play an important role in the immune defense by forming neutrophil extracellular traps (NETs), which are composed of neutrophil-derived DNA together with cytoplasmic, granular, and nuclear proteins [9]. NETs have been shown to play a significant role when it comes to trypsin activation and further recruitment of neutrophils in AP [10].

Accumulating data suggest that the complement system exerts important roles in several diverse processes. In addition to its classical role in microbial defense, complement can recruit neutrophils and regulate inflammation and tissue damage in different inflammatory conditions [11-15]. C3 is the central molecule of complement, positioned at the crossroad of all the complement activation pathways. It has been shown that trypsin can cleave C3 and facilitate leukocyte activation [16]. Deposition of C3 on acinar cells and histological damage of acinar cells were shown to increase in the taurocholate-induced model of AP [11]. In addition, in an endotoxin model of brain injury, C3-deficient mice exhibited decreased neutrophil recruitment and endothelial activation [15]. Proteolytic cleavage of C3 during complement activation or inflammation results in the release of $\mathrm{C} 3 \mathrm{a}$ and $\mathrm{C} 3 \mathrm{~b}$ fragments. Increased levels of C3a are found in early AP patients and the levels are directly correlated with the severity of the disease [17]. Both C3a and C5a are potent anaphylatoxins known to exert numerous proinflammatory effects in the pancreas, including activation and recruitment of neutrophils [18]. C5a has been shown to play both protective and harmful roles in AP [19, 20]. On the other hand, NETs themselves could activate both the classical [21] and the alternative complement pathway [22], and consequently start a self-amplifying loop. However, the role of C3 in NET formation and pathological inflammation in severe AP has not been investigated yet.

Based on the above observations, the aim of this study was to define the functional role of C3 in the generation of NETs as well as tissue inflammation and injury in a model of severe AP in mice.

\section{Karger's}




\section{Materials and Methods}

Animals

All experimental procedures including animals were performed in accordance with the ethical permit approved by the Regional Ethics Committee for animal experimentation at Lund University, Sweden. The C3 gene-deficient mice (B6.129S4-C $3^{\mathrm{tm} 1 \mathrm{Crr}} / \mathrm{J}$ ) were obtained from Jackson Laboratories, and sex and age (9-12 weeks) matched wild-type control mice (C57BL/6J) were obtained from Janvier Labs, Leof Genest-Sant-Isle, France. Animals were fed a standard laboratory diet and given water ad libitum. Mice were anesthetized by intraperitoneal administration with $75 \mathrm{mg}$ of ketamine hydrochloride (Hoffmann-La Roche, Basel, Switzerland) and $25 \mathrm{mg}$ of xylazine (Janssen Pharmaceutica, Beerse, Belgium) per kilogram body weight.

\section{Taurocholate-Induced Pancreatitis}

Mice were anesthetized and a midline incision was performed into the abdominal cavity. The duodenum was identified, and traction sutures was placed $1 \mathrm{~cm}$ proximal and $1 \mathrm{~cm}$ distal of the papilla of Vater in order to immobilize the duodenum. A small puncture was made with a 23-G needle through the duodenal wall opposite the papilla of Vater. A polyethylene catheter connected to a micro-infusion pump (CMA/100, Carnegie Medical, Stockholm, Sweden) was inserted $1 \mathrm{~mm}$ into the common bile duct through the punctured hole, as previously described [23]. A micro clamp was placed on the bile duct close to the liver hilum to prevent hepatic reflux. Infusion of $10 \mu \mathrm{L}$ of $5 \%$ sodium taurocholate (Sigma, St Louis, MO, USA) was then performed into the pancreatic duct for $10 \mathrm{~min}$. After that, the clamp was removed and the duodenal puncture was closed with a purse-string suture. The traction sutures were removed and the abdominal wall was closed with sutures. Sham mice underwent the same procedure except that the pancreatic duct was infused with 10 $\mu \mathrm{L}$ of $0.9 \%$ sodium chloride instead.

Amylase Levels

Blood was obtained from the tail vein and diluted 1:10 in PBS for amylase measurement using a commercially available kit (Reflotron, Roche Diagnostics $\mathrm{GmbH}$, Mannheim, Germany) following the instructions of the manufacturer.

\section{Myeloperoxidase Activity}

Myeloperoxidase (MPO) activity was determined in lung and pancreatic tissue as an indicator of neutrophil infiltration. Small pieces of lung and pancreas were harvested and snap frozen in liquid nitrogen. The pieces were later thawed, weighed, and homogenized for $1 \mathrm{~min}$ in a 1-mL mixture of PBS and aprotinin (10,000 kallekrein inactivator units per milliliter; Trasylol, Bayer HealthCare AG, Leverkusen, Germany). The homogenates were centrifuged for $10 \mathrm{~min}$ at $15,300 \mathrm{~g}$ at $4{ }^{\circ} \mathrm{C}$. Supernatants were removed and stored at $-20^{\circ} \mathrm{C}$. The pellets were mixed with $1 \mathrm{~mL}$ of $0.5 \%$ hexadecyltrimethylammonium bromide and frozen for $24 \mathrm{~h}$, after which they were thawed and sonicated for $90 \mathrm{~s}$. The pellets were then placed in a water bath $\left(60^{\circ} \mathrm{C}\right.$ for $2 \mathrm{~h}$ ) and MPO activity was quantified spectrophotometrically as the MPO catalyzed change in absorbance during the redox reaction of $\mathrm{H}_{2} \mathrm{O}_{2}\left(450 \mathrm{~nm}\right.$, with reference filter $\left.540 \mathrm{~nm}, 25^{\circ} \mathrm{C}\right)$ as previously described [24].

\section{Measurement of DNA-Histone Complexes}

Circulating levels of DNA-histone complexes were determined in blood obtained from the inferior vena cava. Blood was diluted (1:10) in acid citrate dextrose and centrifuged for $5 \min \left(15,300 \mathrm{~g}, 4^{\circ} \mathrm{C}\right)$. DNA-histone complexes were also determined in the supernatants of in vitro experiments. Briefly, neutrophils were isolated from bone marrow as described previously [25]. A total of $1 \times 10^{6}$ neutrophils was stimulated with PMA (phorbol 12-myristate 13-acetate; $0.3 \mu \mathrm{g} / \mathrm{mL}$, cat. No. P1585, Sigma-Aldrich) or mouse recombinant C3a (1.0 $\mu \mathrm{g} / \mathrm{mL}$, cat. No. 8085-C3-025, R\&D Systems Europe, UK) in suspension. Samples were centrifuged $(400 \mathrm{~g}, 5 \mathrm{~min})$ and supernatants were collected for the measurement of DNA-histone complexes. A Cell Death Detection Elisa Plus kit (Roche Diagnostics, Mannheim, Germany) was used to quantify DNA-histone complexes according to the manufacturer's instructions.

\section{Enzyme-Linked Immunosorbent Assay}

Levels of CXCL1 and CXCL2 in pancreatic tissue were measured in stored supernatants obtained from homogenized tissue. Double-antibody ELISA kits (R\&D Systems Europe, Abingdon, Oxon, UK) were used according to the manufacturers' instructions. For measuring plasma levels of CXCL2, matrix metallopeptidase-9 (MMP-9) and IL-6, commercially available ELISA kits (R\&D Systems Europe) were used according to the manu-

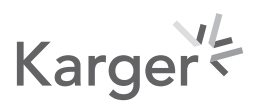


facturer's instructions. The blood used for this was collected from the inferior vena cava and diluted (1:10) in acid citrate dextrose, centrifuged $\left(15,300 \mathrm{~g}\right.$ for $5 \mathrm{~min}$ at $\left.4{ }^{\circ} \mathrm{C}\right)$, and then plasma was stored at $-20^{\circ} \mathrm{C}$ until use.

Histology

For histological analysis, pancreatic heads were fixed in $4 \%$ formaldehyde phosphate buffer overnight, after which they were dehydrated and paraffin embedded. Six-micrometer sections were prepared using a microtome and then stained with hematoxylin and eosin. Images were taken using a ScanScope slide scanner. A preexisting scoring system was used in a blinded manner. The scoring system includes edema, acinar cell necrosis, hemorrhage, and neutrophil infiltration on a 0 (absent) to 4 (extensive) scale, as described in detail previously [26].

\section{Electron Microscopy}

Deparaffinized pancreatic tissue samples were fixed for $30 \mathrm{~min}$ at room temperature using 2.5\% glutaraldehyde in $0.15 \mathrm{~mol} / \mathrm{L}$ sodium cacodylate, pH 7.4 cacodylate buffer), and used for detection of NETs. After fixation the samples were washed with cacodylate buffer and dehydrated with an ascending ethanol series (10 min/step) from 50\% (vol/vol) to absolute ethanol. Specimens were subjected to critical-point dying in carbon dioxide, with absolute ethanol as the intermediate solvent. The specimens were examined using a Jeol/FEI XL 30 FEG at the Core Facility for Integrated Microscopy at the Panum Institute (University of Copenhagen, Denmark). The location of individual target molecules was analyzed at high resolution by ultrathin sectioning and transmission immunoelectron microscopy. The coverslips with specimens were embedded in Epon 812 and sectioned with a diamond knife in an ultramicrotome into 50-nm-thick ultrathin sections. Sections were incubated over night at $4{ }^{\circ} \mathrm{C}$ with gold-labelled primary antibodies against elastase (ab68672, Abcam, Cambridge, UK) or citrullinated histone 3 (ab5103, Abcam, Cambridge, UK) for immunohistochemistry. For quantification of NET areas, characteristic web-like fibrillar structures were first identified at high magnification. The identified NET areas were further confirmed in combination with TEM/gold-labelled immunostaining for NET markers. Quantitative NETs surface area assessment was performed using Adobe Photoshop CS5. In short, the number of pixels/square micrometer was determined by using the Ruler Tool. NET areas were then translated into pixel numbers with the Magic Wand Selection Tool. Thus, the fractions of the NET area relative to the entire area of a given electron micrograph was calculated.

\section{Flow Cytometry}

Neutrophils were isolated from bone marrow and stimulated with PMA or recombinant C3a in suspension as described above. Samples were centrifuged ( $400 \mathrm{~g}, 5 \mathrm{~min}$ ) and pellets containing neutrophils were fixed with $2 \%$ formaldehyde. After washing twice with PBS containing 2\% FBS, cells were incubated with the following primary antibodies: phycoerythrin (PE) conjugated anti-Ly6G (clone 1A8, BD Pharmingen), fluorescein isothiocyanate (FITC) conjugated anti-MPO antibody (mouse: ab90812 Abcam, Cambridge, MA, USA) and rabbit anti-citH3 (citrulline 2,8,17, ab5103; Abcam) in PBS containing 5\% donkey serum. After washing twice, cells were incubated with rat anti-rabbit allophycocyanin (APC) conjugated secondary antibody (A-21038, Thermo Fisher Scientific, Rockford, IL, USA). Flow cytometry analysis was performed using a CytoFLEX flow cytometer (Becton Dickinson, Mountain View, CA, USA). Neutrophils were defined as $\mathrm{Ly}_{6 \mathrm{G}}{ }^{+}$cells. MPO and citrullinated $\mathrm{H} 3$ were determined on gated $\mathrm{Ly}_{6} \mathrm{G}^{+}$cells. Quadrant gates were set using florescence minus one (FMO) controls.

\section{Western Blot}

For confirmation of the C3 deficiency in protein level (online suppl. Fig. 1; for all online suppl. material, see www.karger.com/doi/10.1159/000513845), liver homogenate samples were run on a 10\% SDS-PAGE, transferred to PVDF membranes and blotted for C3 using goat anti-mouse C3 (cat. No. 55500, ICN Biomedicals, USA), then detected using rabbit anti-goat HRP-labeled secondary antibody (cat No. P0449, DAKO, Santa Clara, CA, USA), and developed using enhanced chemiluminescence reagent (Millipore) and imaged using a Chemidoc CCD camera (BioRad, Hercules, CA, USA). Beta-actin used as a loading control was detected with monoclonal antibody from Abcam (cat No. ab8226).

\section{Genotyping}

For confirmation of the C3 deficiency at a genetic level (online suppl. Fig. 1), genotyping PCR was carried out using common forward primer ATCTTGAGTGCACCAAGCC and either WT-specific reverse primer GGTTGCAGCAGTCTATGAAGG or KO-specific primer GCCAGAGGCCACTTGTGTAG, in combination with RedTaq PCR ReadyMix (Sigma-Aldrich), according to manufacturer's instructions.

\section{Karger's}




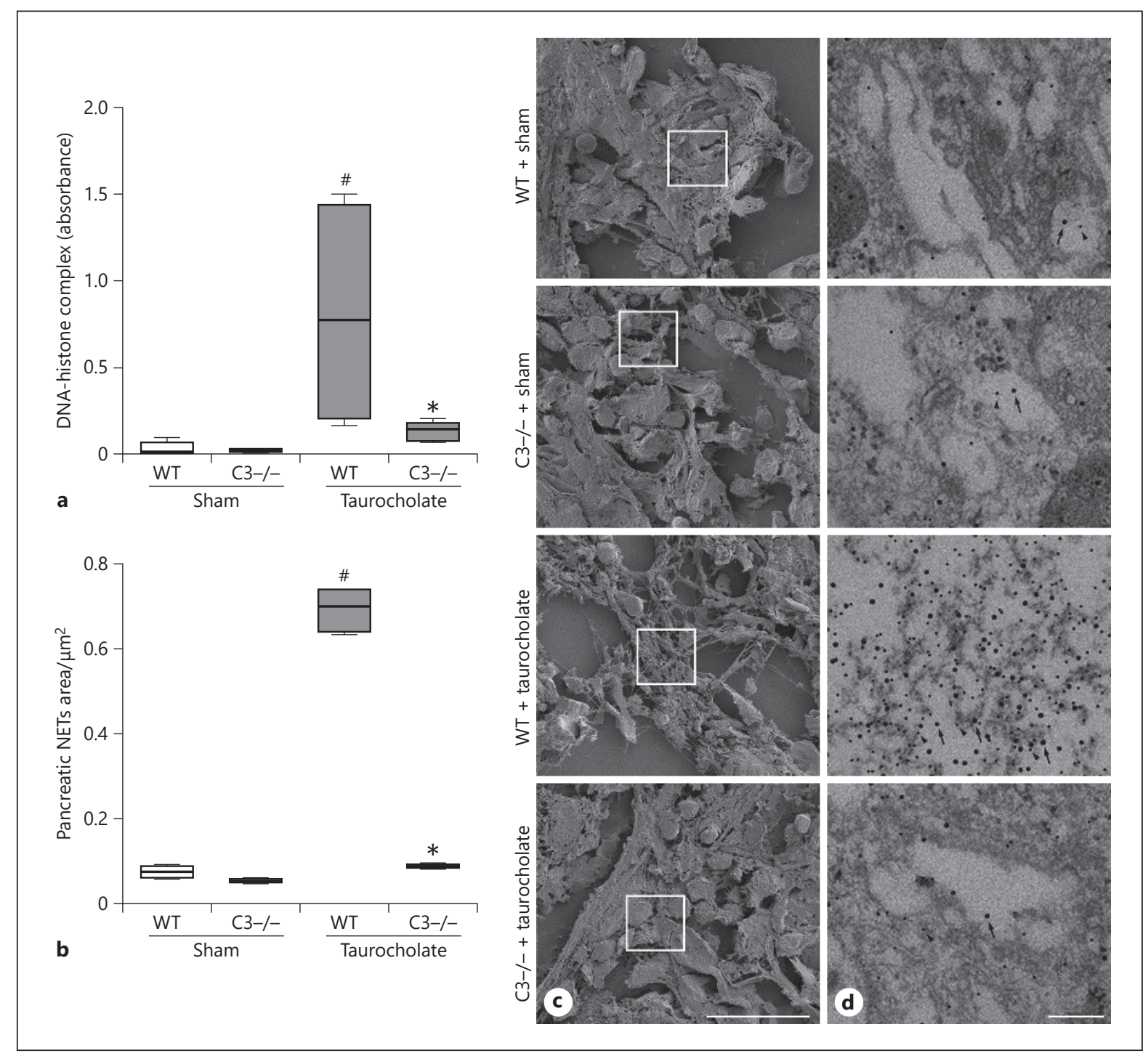

Fig. 1. a Plasma levels of DNA-histone complexes. b Aggregate data on NET formation in the pancreas. c Scanning electron microscopy showing extracellular web-like structures consistent with NETs in the pancreas of mice (wild-type or C3-deficient) exposed to taurocholate or saline. Scale bar, $5 \mu \mathrm{m}$. $\mathbf{d}$ Transmission electron microscopy of the indicated area of interest from $\mathbf{c}$ incubated with gold-labeled anti-citrullinated histone 3 (large gold particles, arrows) and anti-elastase (small gold particles, arrowheads) antibodies; $n=$ 4. Scale bar, $0.25 \mu \mathrm{m}$. Pancreatitis was induced by infusion of sodium taurocholate (5\%) into the pancreatic duct in wild-type and C3-deficient mice. Control mice were infused with saline alone. Samples were collected $24 \mathrm{~h}$ after the induction of pancreatitis. The boxes represent the median (25-75 percentile) and the whiskers extend from the minimum to the maximum values; $n=4-5$. ${ }^{\#} p<0.05$ versus WT Sham; * $p<$ 0.05 versus WT taurocholate.

\section{Statistical Analyses}

Data analysis was conducted using GraphPad Prism 8. Data are presented in box plots (25-75 percentiles) where the horizontal line indicates the median of the group and the whiskers extend from the minimum to the maximum values. Statistical evaluations were performed using nonparametric tests (Mann-Whitney). $p<0.05$ was considered significant, and $n$ represents the number of animals or independent experiments in each group. 
Fig. 2. Quantitative measurements of blood amylase levels. Pancreatitis was induced by retrograde infusion of sodium taurocholate $(5 \%)$ into the pancreatic duct in wild-type and C3-deficient mice. Control mice were infused with saline alone. Samples were collected $24 \mathrm{~h}$ after the induction of pancreatitis. The boxes represent the median (25-75 percentile) and the whiskers extend from the minimum to the maximum levels; $n=4-5$. ${ }^{\#} p<0.05$ versus WT Sham; $* p<0.05$ versus WT taurocholate.

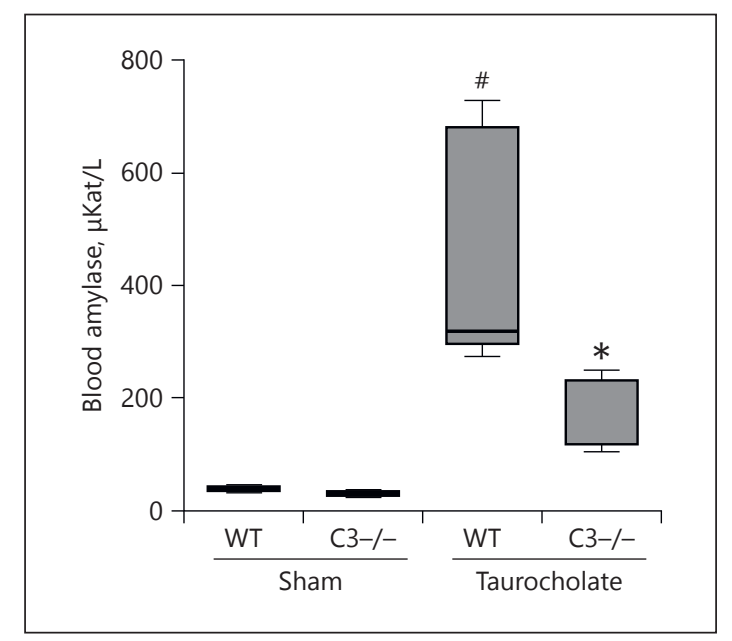

\section{Results}

C3 Is Required for NETs Generation in the Inflamed Pancreas

Infusion of taurocholate increased plasma levels of DNA-histone complexes by 16-fold compared to sham-treated animals (Fig. 1a). In the C3-deficient group, the levels of DNAhistone complexes in the pancreas were reduced by $89 \%$ compared to wild-type (Fig. 1a). This observation was confirmed by experiments revealing that in wild-type animals, infusion of taurocholate increased the generation of extracellular fibrillar and web-like structures (Fig. 1c) that colocalized with neutrophil-derived granule protein elastase as well as with citrullinated histone 3 (Fig. 1d), indicating the presence of NETs in the inflamed pancreas. Notably, in the C3-deficient group formation of NETs was reduced by 97\% (NETs area in the field) compared to wild-type in the taurocholate-provoked pancreas (Fig. 1b), indicating that C3 is involved in the generation of NETs in the pancreas. C3-deficiency was confirmed by Western blotting and genotyping (online suppl Fig. 1).

\section{C3-Deficient Mice Had Less Organ Injury in AP Compared to Wild-Type}

Retrograde infusion of taurocholate in the pancreatic duct increased blood levels of amylase by 12-fold compared to sham-treated animals (Fig. 2). In the C3-deficient mice, the increase in taurocholate-mediated induction of blood amylase levels was significantly reduced by $65 \%$ compared to wild-type (Fig. 2). Injection of saline into the pancreatic duct did not elevate the levels of blood amylase in either wild-type or C3-deficient mice (Fig. 2). Examination of tissue morphology revealed that taurocholate infusion caused destruction of the microarchitecture in the pancreas exemplified by acinar cell necrosis, hemorrhage, edema formation, and leukocyte infiltration (Fig. 3a-e). When taurocholate was infused in the C3-deficient mice, substantially less tissue injury was observed compared to wild-type (Fig. 3a-e). Quantification of taurocholate-induced issue damage revealed that in the C3-deficient animals there were 67\% less edema (Fig. 3b), 72\% less hemorrhage (Fig. 3c), 66\% less acinar cell injury (Fig. 3d), and 70\% less neutrophil infiltration (Fig. 3e) in the inflamed pancreas compared with wild-type mice.

Less Neutrophil Recruitment in the Inflamed Pancreas of the C3-Deficient Mice

MPO activity in the pancreas increased by 20 -fold after infusion of taurocholate (Fig. 4a) compared to sham-treated animals. In the C3-deficient mice taurocholate-induced activity of 


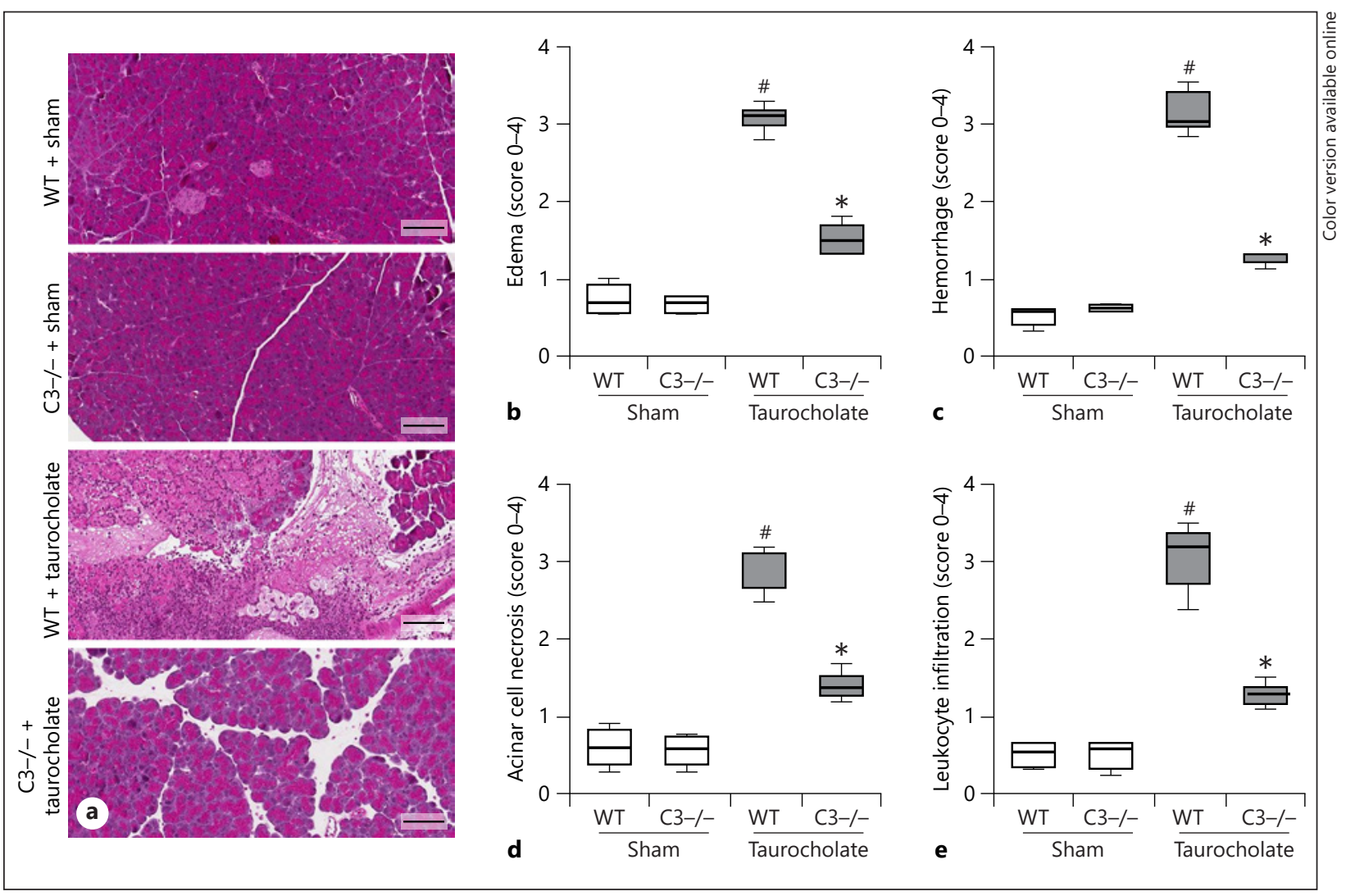

Fig. 3. a Representative hematoxylin and eosin sections of the head of the pancreas from indicated groups. Scale bars, $100 \mu \mathrm{m}$. Histological quantification of edema (b), hemorrhage (c), acinar cell necrosis (d), and leukocyte infiltration (e). Pancreatitis was induced by retrograde infusion of sodium taurocholate (5\%) into the pancreatic duct in wild-type and C3-deficient mice. Control mice were infused with saline alone. Samples were collected $24 \mathrm{~h}$ after the induction of pancreatitis. The boxes represent the median (25-75 percentile) and the whiskers extend from the minimum to the maximum levels; $n=4-5$. ${ }^{*} p<0.05$ versus WT Sham; $* p<0.05$ versus WT taurocholate.

MPO in the pancreas decreased by $74 \%$ compared to taurocholate-treated wild-type mice (Fig. 4a), corresponding well to the reduction (by 68\%) of the number of neutrophils in the inflamed pancreas of the C3-deficient mice (Fig. 3e). Additionally, challenge with taurocholate increased pancreatic levels of CXCL1 and CXCL2 (Fig. 4b, c). In the C3-deficient mice the pancreatic levels of CXCL1 and CXCL2 were decreased by 83 and 97\%, respectively, compared to wild-type mice (fig. 4b, c). Administration of saline had no impact on levels of MPO, CXCL1, and CXCL2 in the pancreas (Fig. $4 a-c)$.

\section{Reduced Pulmonary Neutrophilia in the C3-Deficient Mice}

Accumulation of neutrophils in the lung is part of a systemic inflammatory response in severe AP and we observed that infusion of taurocholate in wild-type mice increased lung levels of MPO by 30-fold compared to sham-treated animals (Fig. 5a). In the C3-deficient mice the lung levels of MPO were 77\% lower after infusion of taurocholate compared to wild-type mice (Fig. 5a). Moreover, taurocholate infusion increased CXCL2, MMP-9, and IL-6 levels in the plasma by 85-, 7-, and 28-fold in wild-type animals, respectively (Fig. 5b-d). Taurocholate-induced increased plasma levels of CXCL2, MMP-9, and IL-6 


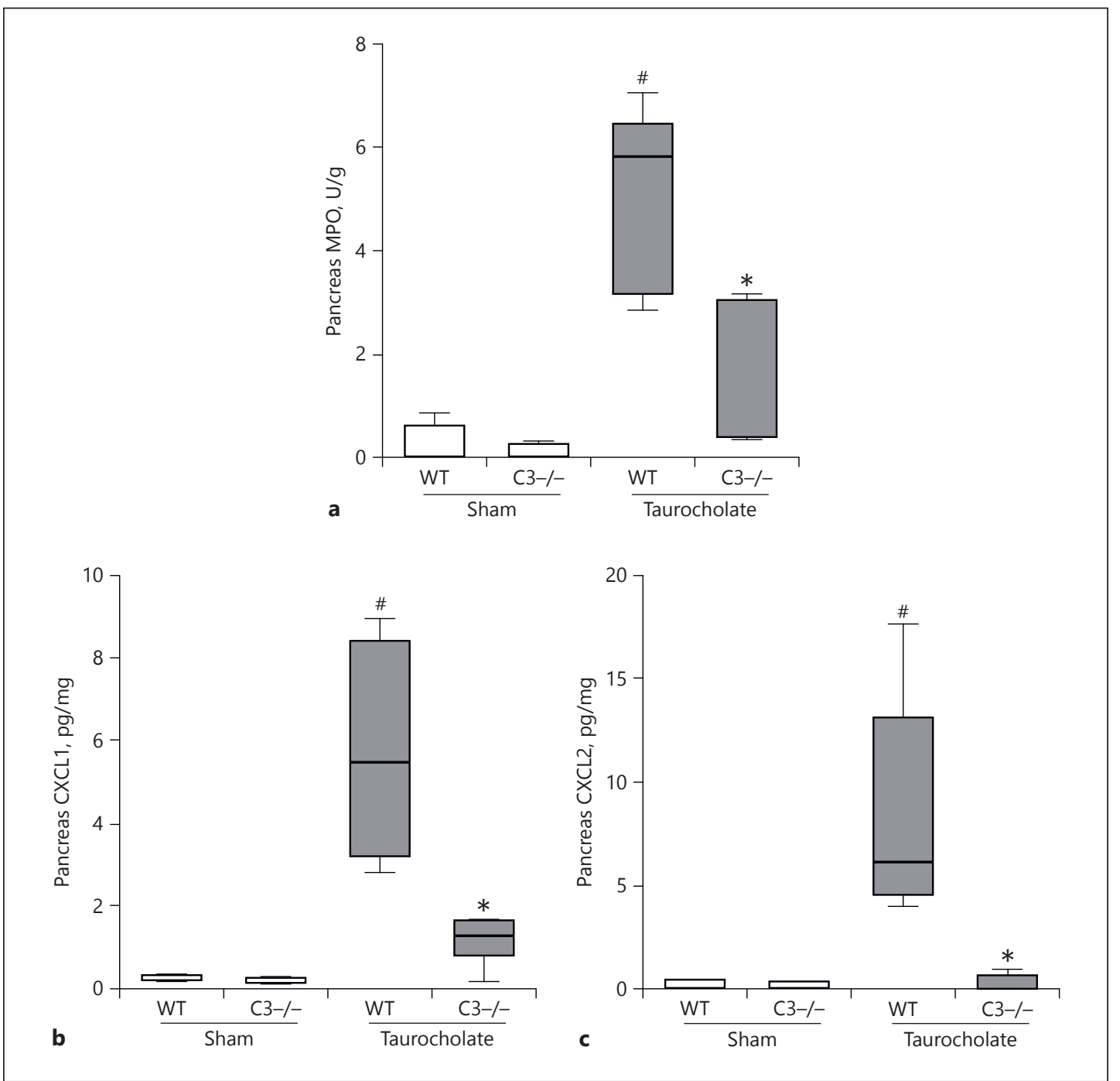

Fig. 4. Quantitative measurements of pancreatic levels of MPO (a), CXCL1 (b) and CXCL2 (c). Pancreatitis was induced by retrograde infusion of sodium taurocholate (5\%) into the pancreatic duct in wild-type and C3-deficient mice. Control mice were infused with saline alone. Samples were collected $24 \mathrm{~h}$ after the induction of pancreatitis. The boxes represent the median (25-75 percentile) and the whiskers extend from the minimum to the maximum levels; $n=4-5$. ${ }^{\#} p<0.05$ versus WT Sham; ${ }^{*} p<0.05$ versus WT taurocholate.

were decreased by 92,83 , and 53\% in animals lacking C3 compared to wild-type mice (Fig. 5b-d).

Neutrophils from Wild-Type and C3 Gene-Deficient Mice Have Similar NET-Forming Ability

To evaluate whether neutrophils from C3-deficent mice have a similar ability to form NETs as the wild-type neutrophils, we performed a separate in vitro experiment. Briefly, neutrophils from the bone marrow of wild-type or C3-deficient mice were incubated with PBS or PMA and levels of NETs formation were similar in both wild-type and C3-deficient mice (Fig. 6a), indicating that there is no intrinsic faults in the neutrophils of C3 gene-deficient mice in terms of NET-forming ability. Similar results were observed when MPO and citrullinated histone 3 expression were evaluated on neutrophils by flow cytometry (Fig. 6b, c). 


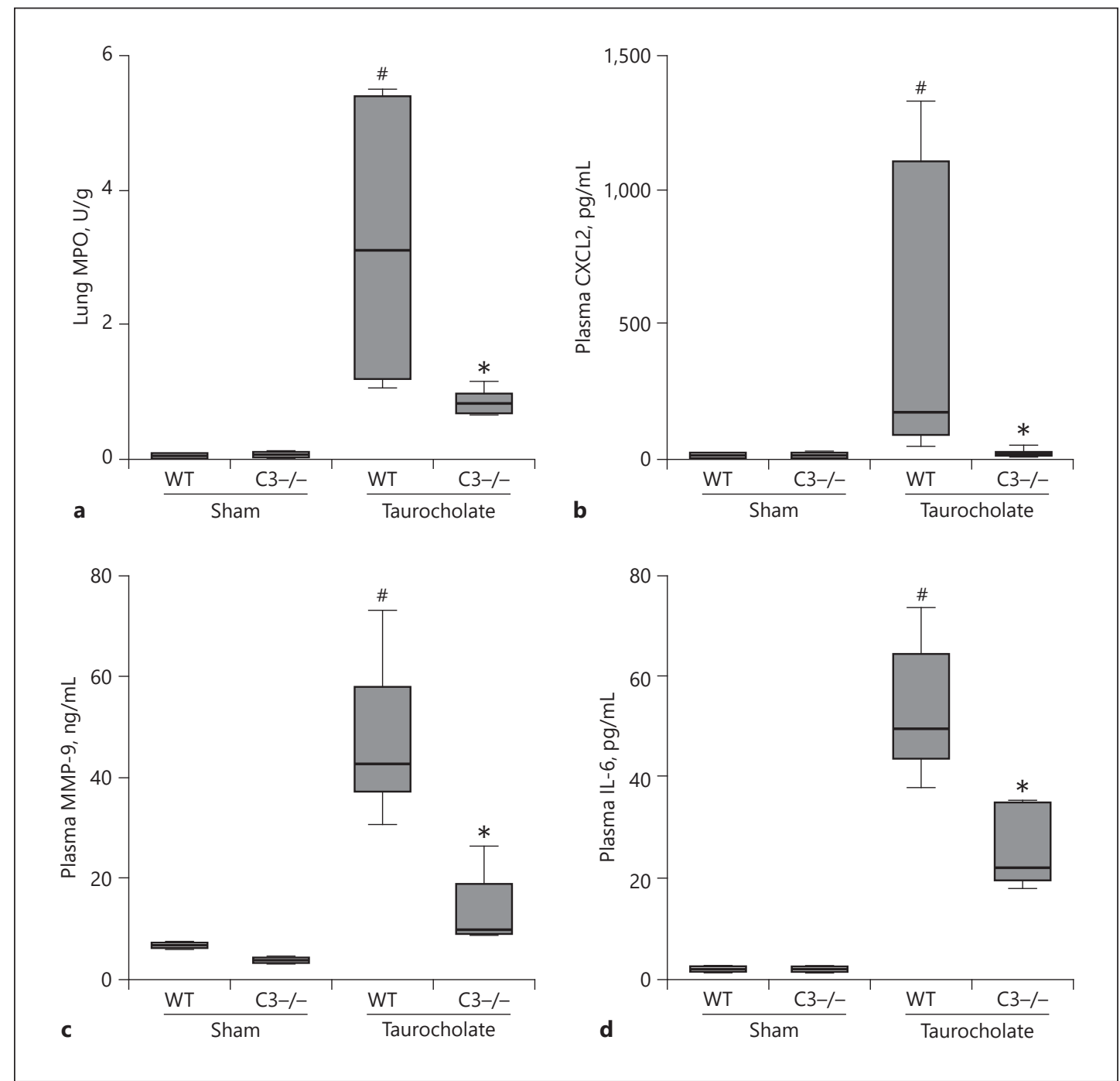

Fig. 5. a Pulmonary levels of MPO. Plasma levels of CXCL2 (b), MMP-9 (c), and IL-6 (d). Pancreatitis was induced by retrograde infusion of sodium taurocholate (5\%) into the pancreatic duct in wild-type and C3-deficient mice. Control mice were infused with saline alone. Samples were collected $24 \mathrm{~h}$ after the induction of pancreatitis. The boxes represent the median (25-75 percentile) and the whiskers extend from the minimum to the maximum levels; $n=4-5$. ${ }^{\#} p<0.05$ versus WT Sham; ${ }^{*} p<0.05$ versus WT taurocholate.

\section{C3a Alone Is Not Sufficient to Induce NETs Formation}

To determine whether the C3 cleavage product, C3a, can directly regulate NET formation, bone marrow neutrophils from wild-type mice were exposed to recombinant C3a $(1.0 \mu \mathrm{g} / \mathrm{mL})$ or PMA $(0.3 \mu \mathrm{g} / \mathrm{mL})$ for $3 \mathrm{~h}$ at $37^{\circ} \mathrm{C}$. Challenge with recombinant C3a had no effect on NETs formation by neutrophils (Fig. 7a). In contrast, incubation of neutrophils with PMA induced NET formation (Fig. 7a). In addition, we evaluated expression of MPO and citrullinated histone 3, a specific marker of NETs, on neutrophils by flow cytometry. PMA stimulation increased MPO and citrullinated histone 3-positive neutrophils (Fig. 7b) but recombinant $\mathrm{C} 3 \mathrm{a}$ had no effect on neutrophil expression of MPO and citrullinated histone 3, suggesting that $\mathrm{C} 3 \mathrm{a}$ alone is not sufficient to induce NETs formation in neutrophils. 


\begin{tabular}{ll} 
European & \multicolumn{2}{l}{ Eur Surg Res 2020;61:163-176 } \\
\cline { 2 - 2 } $\begin{array}{l}\text { Sul: 10.1159/000513845 } \\
\text { Surgical Research }\end{array}$ & $\begin{array}{l}\text { ○ 2021 The Author(s). Published by S. Karger AG, Basel } \\
\text { www.karger.com/esr }\end{array}$ \\
\cline { 2 - 2 } & Linders et al.: Complement Component 3 and Acute Pancreatitis
\end{tabular}

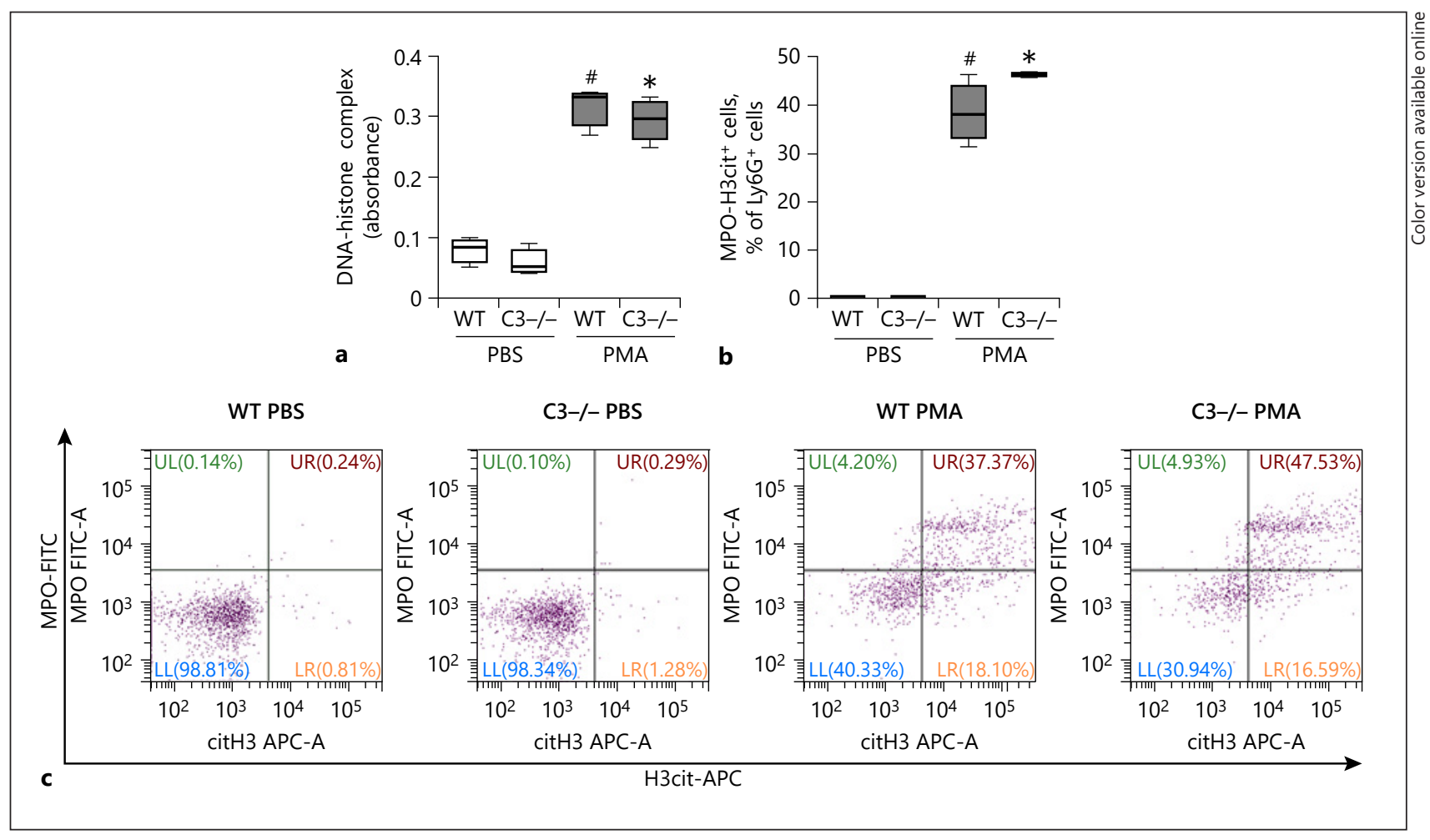

Fig. 6. Comparison of the NET-forming ability of neutrophils from wild-type and C3 gene-deficient mice. Neutrophils isolated from the bone marrow of wild-type or C3-deficient mice were stimulated with PMA (0.3 $\mu \mathrm{g} / \mathrm{mL}$ ) or with PBS alone in suspension and analyzed by ELISA and flow cytometry. a Levels of DNA-histone complexes in supernatants. b MPO and citrullinated histone 3 on neutrophils (defined as Ly6G ${ }^{+}$cells). c Representative dot plots showing the presence of MPO and citrullinated histone 3 on neutrophils. The boxes represent the median (25-75 percentile) and the whiskers extend from the minimum to the maximum levels; $n=4{ }^{*} p<0.05$ versus WT PBS; ${ }^{*} p<0.05$ versus C3-deficient PBS.

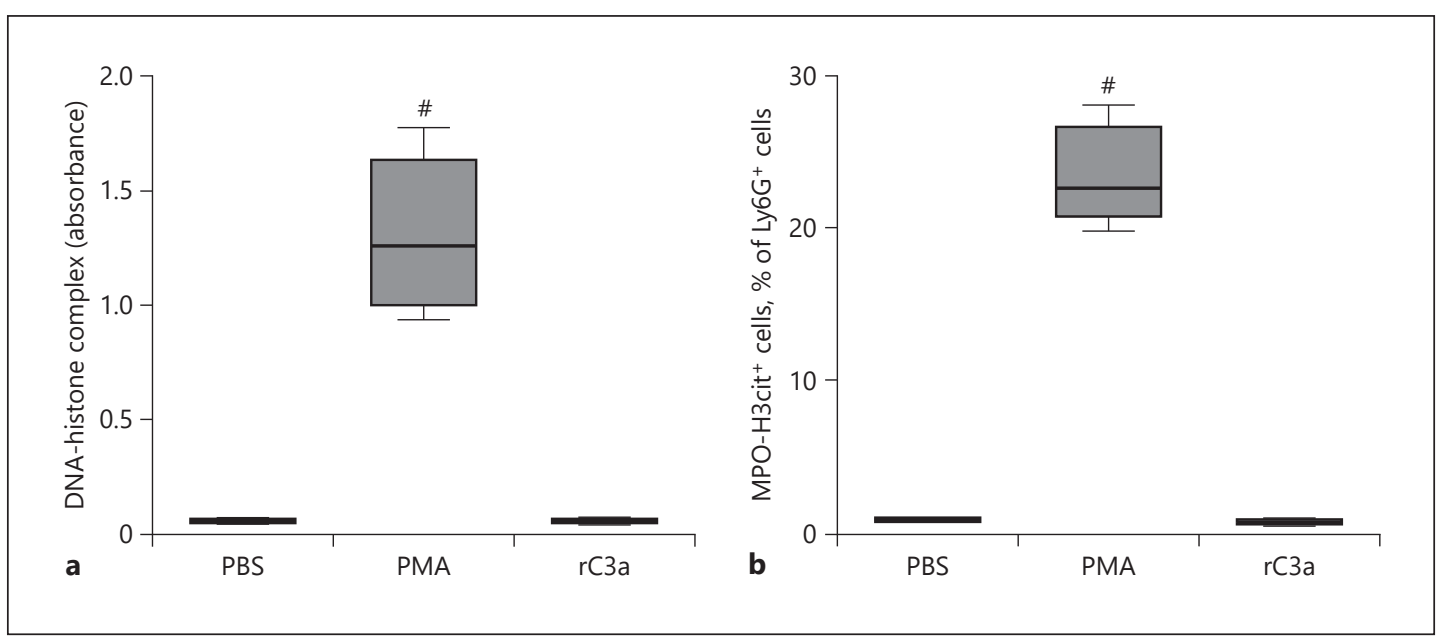

Fig. 7. NETs formation in vitro. Neutrophils isolated from the bone marrow of wild-type mice were stimulated with recombinant C3a $(1 \mu \mathrm{g} / \mathrm{mL})$, PMA $(0.3 \mu \mathrm{g} / \mathrm{mL})$, or with PBS alone in suspension and analyzed by ELISA and flow cytometry. a Levels of DNA-histone complexes in supernatants. b MPO and citrullinated histone 3 on neutrophils (defined as Ly $6 \mathrm{G}^{+}$cells). The boxes represent the median (25-75 percentile) and the whiskers extend from the minimum to the maximum levels; $n=4$. ${ }^{*} p<0.05$ versus WT PBS. 
Fig. 8. Schematic illustration showing complement component 3-mediated NET formation and pancreatic tissue damage in AP.

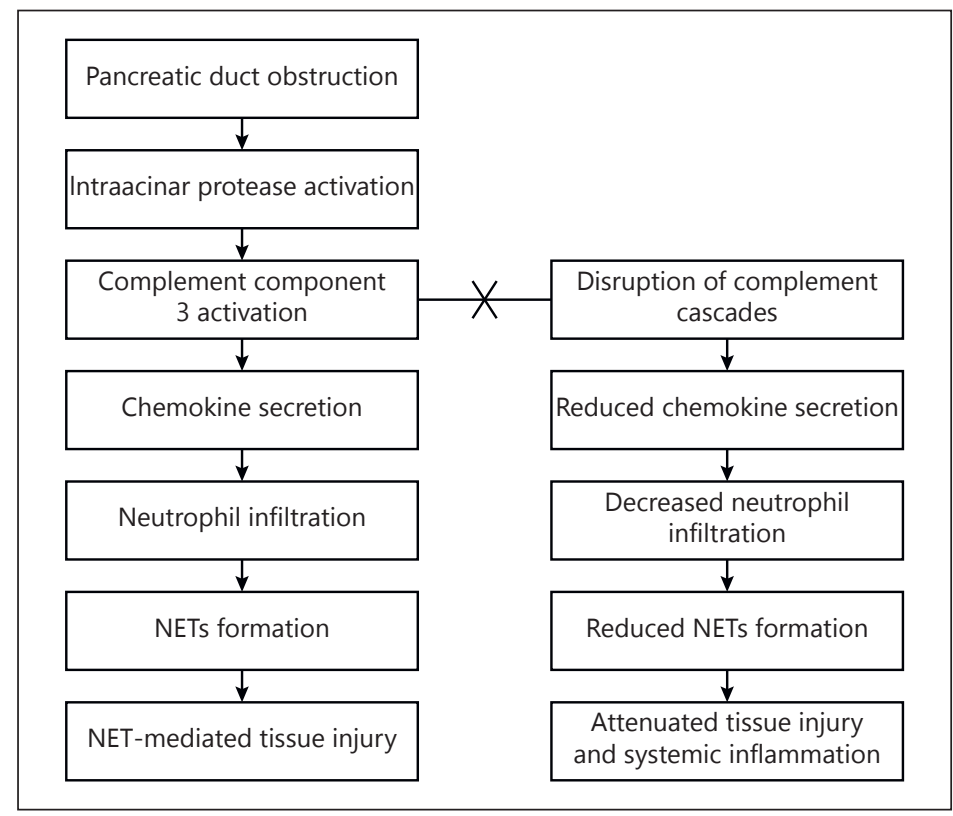

\section{Discussion}

Our data show for the first time a crucial role of C3 in the development of severe AP. These results demonstrate that $\mathrm{C} 3$ is a potent contributor to pancreatic tissue damage, systemic inflammation, neutrophil recruitment, and ensuing NETs formation in the inflamed pancreas. Our findings suggest an important role of C3 in the inflamed pancreas and that targeting C3 activation by drugs such as by compstatin [27] might be an effective way to inhibit inflammatory processes in AP.

The complement system constitutes a significant component in the innate immune system [14]. Early findings of decreased serum levels of C3 and C4 during AP suggested complement consumption and a potential role of complement mediating local and systemic inflammation [28]. A more recent study reported increased plasma levels of C3a and the terminal complement complex (sC5b-9) and that levels of these complement products correlate with disease severity in patients with AP [17]. Although there are very few direct studies on the functional role of complement in AP one study showed that trypsin is capable of cleaving and activating central complement components, leading to formation of C3a, implicating complement in the development of AP [29]. The literature on the role of C5a is complex. One study reported that C5-deficient mice develop significantly less tissue edema [19] whereas another study observed increased edema formation in AP [20]. This discrepancy might be related to use of different experimental models to induce AP. Herein, we observed that tissue damage (necrosis, edema and hemorrhage) in severe AP was significantly decreased in mice lacking C3 and hence also having lower levels of C5a, which is generated in the next step of the complement cascade. Moreover, our results showed that amylase levels were reduced by $65 \%$ in $\mathrm{AP}$ animals deficient of $\mathrm{C} 3$, further suggesting that $\mathrm{C} 3$ plays an important role in mediating organ damage in the inflamed pancreas. These data constitute the first evidence in the literature that C3 is involved in the development of AP.

It is generally held that neutrophil infiltration is a key component in mediating tissue damage in severe AP $[3,7,8]$. In the present study, we found that taurocholate challenge elevated MPO activity and the number of extravascular neutrophils in the inflamed pancreas. 
Taurocholate-induced MPO levels and the number of tissue neutrophils in the pancreas were markedly reduced in C3-deficient mice, suggesting that C3 is an important regulator of neutrophilia in the inflamed pancreas. Neutrophil trafficking to sites of inflammation is also orchestrated by secreted CXC chemokines, including CXCL1 and CXCL2 [5, 6], and a functional role of CXC chemokines has been documented in AP [6]. We observed that taurocholate caused a clear-cut increase in CXCL1 and CXCL2 levels in the pancreas. Taurocholateinduced pancreatic levels of CXCL1 and CXCL2 were significantly attenuated in C3 gene-deficient mice, suggesting that C3 is a critical regulator of chemokines secretion in AP. Moreover, the effect of disrupted C3 on the recruitment of neutrophil might help to explain the protective effect of NET-mediated tissue damage in C3-deficient AP mice (Fig. 8). Recent findings have shown that neutrophil-derived NETs exert an important role in the pathophysiology of AP by triggering activation of trypsinogen and tissue inflammation in the pancreas [10]. In addition, a recent study has shown that a cold-inducible RNA-binding protein (eCIRP), a novel damage-associated molecular pattern molecule, is present in NETs and that NET-associated eCIRP can cause tissue damage in AP [30]. Furthermore, other studies indicated that NET formation is regulated by the c-Abelson (c-Abl) kinase [31] and the histone-citrullinating enzyme peptidylarginine deiminase 4 (PAD4) in AP [32]. Knowing that the C3 activation product, $\mathrm{C} 3 \mathrm{a}$, exerts proinflammatory functions, we evaluated whether $\mathrm{C} 3 \mathrm{a}$ alone can induce histone 3 citrullination and NETs formation in bone marrow-derived neutrophils. Our data indicate that recombinant $\mathrm{C} 3 \mathrm{a}$ alone is not sufficient to induce histone 3 citrullination and NETs formation. It should be noted that other alternative mechanisms involving C3 could be relevant in NET formation and should be a subject of future studies. In addition, by use of electron microscopy it was shown that taurocholate-induced formation of NETs was markedly reduced in the pancreas of animals lacking C3. Considering the key role of neutrophils in the development of pancreatitis $[3,7,10]$, it might suggest that decreased recruitment of neutrophils could help to explain the tissue-protective effect in pancreatitis animals lacking C3.

Severe pancreatitis is associated with systemic inflammation and remote organ injury [7]. A hallmark of systemic complications in severe AP is the pulmonary infiltration of neutrophils [8]. Herein, we observed that pulmonary levels of MPO was markedly increased in response to taurocholate challenge. Interestingly, a taurocholate-induced increase of pulmonary activity of MPO was significantly reduced in C3-deficient animals, suggesting that C3 also controls remote accumulation of neutrophils in the lung in severe AP. This notion is supported by a previous study reporting that injection of AP serum into lungs caused pulmonary infiltration of neutrophils, which was prevented by treatment with soluble complement receptor type 1 [33]. In this context, it is also interesting to note that complement activation with increased levels of C3a was observed only in rats with severe AP but not mild $\mathrm{AP}$, further implicating C3 in severe systemic inflammation [34]. The notion that C3 regulates systemic inflammation is also in line with our observation that the taurocholate-induced elevations of circulating plasma levels of IL-6, CXCL2, and MMP-9 were greatly suppressed in mice deficient of C3.

In conclusion, our study suggests that $\mathrm{C} 3$ regulates important aspects of the pathophysiology of AP. In particular, C3 was found to play an important role in neutrophil recruitment and ensuring NETs formation in the inflamed pancreas. Moreover, our findings also suggest that C3 exert a role in systemic inflammation and pulmonary neutrophilia in severe AP. Thus, this study not only delineates potential mechanisms of C3-dependent inflammation in the pancreas but also suggests that pharmacological targeting of C3 function might be a useful strategy to antagonize local tissue damage and remote organ dysfunction in patients with severe AP.

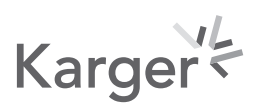


Linders et al.: Complement Component 3 and Acute Pancreatitis

\section{Statement of Ethics}

All experimental procedures including animals were performed in accordance with the ethical permit (Dnr: 5.2.18-4897/16) approved by the Regional Ethics Committee for animal experimentation at Lund University, Sweden. The ARRIVE guidelines were consulted for all animal experiments.

\section{Conflict of Interest Statement}

The authors have no conflicts of interest to declare.

\section{Funding Sources}

This work was supported by funding from the Swedish Research Council (537-2014-341) and Einar and Inga Nilsson foundation. Raed Madhi is supported by a doctoral fellowship from Misan University, College of Science, Iraq.

\section{Author Contributions}

J.L., R.M., B.C.K., M.M. performed experiments, interpreted results, and contributed to the writing. A.M.B. interpreted results and contributed to the writing. M.R. conceived and designed the study and contributed to the writing. All authors approved the final manuscript.

\section{References}

1 Kadiyala V, Suleiman SL, McNabb-Baltar J, Wu BU, Banks PA, Singh VK. The Atlanta Classification, Revised Atlanta Classification, and Determinant-Based Classification of Acute Pancreatitis: which is best at stratifying outcomes? Pancreas. 2016 Apr;45(4):510-5.

2 Bhatia M, Wong FL, Cao Y, Lau HY, Huang J, Puneet P, et al. Pathophysiology of acute pancreatitis. Pancreatology. 2005;5(2-3):132-44.

3 Regnér S, Manjer J, Appelros S, Hjalmarsson C, Sadic J, Borgström A. Protease activation, pancreatic leakage, and inflammation in acute pancreatitis: differences between mild and severe cases and changes over the first three days. Pancreatology. 2008;8(6):600-7.

4 van Acker GJ, Perides G, Steer ML. Co-localization hypothesis: a mechanism for the intrapancreatic activation of digestive enzymes during the early phases of acute pancreatitis. World J Gastroenterol. 2006 Apr;12(13): 1985-90.

5 Zhang XW, Liu Q, Wang Y, Thorlacius H. CXC chemokines, MIP-2 and KC, induce P-selectin-dependent neutrophil rolling and extravascular migration in vivo. Br J Pharmacol. 2001 Jun;133(3):413-21.

6 Bhatia M, Hegde A. Treatment with antileukinate, a CXCR2 chemokine receptor antagonist, protects mice against acute pancreatitis and associated lung injury. Regul Pept. 2007 Jan;138(1):40-8.

7 Abdulla A, Awla D, Thorlacius H, Regnér S. Role of neutrophils in the activation of trypsinogen in severe acute pancreatitis. J Leukoc Biol. 2011 Nov; 90(5):975-82.

8 Merza M, Wetterholm E, Zhang S, Regner S, Thorlacius H. Inhibition of geranylgeranyltransferase attenuates neutrophil accumulation and tissue injury in severe acute pancreatitis. J Leukoc Biol. 2013 Sep;94(3):493502.

9 Castanheira FV, Kubes P. Neutrophils and NETs in modulating acute and chronic inflammation. Blood. 2019 May;133(20):2178-85.

10 Merza M, Hartman H, Rahman M, Hwaiz R, Zhang E, Renström E, et al. Neutrophil Extracellular Traps Induce Trypsin Activation, Inflammation, and Tissue Damage in Mice With Severe Acute Pancreatitis. Gastroenterology. 2015 Dec;149(7):1920-1931.e8.

11 Seelig R, Lankisch PG, Koop H, Winckler K, Kaboth U, Seelig HP. Complement system in sodium taurocholate pancreatitis in the rat. Res Exp Med (Berl). 1978 Dec;174(1):57-65.

12 Horn JK, Ranson JH, Ong R, Poulis D, Perez HD, Goldstein IM. Complement catabolism and chemotaxis in acute pancreatitis. J Surg Res. 1982 Jun;32(6):569-75.

13 Hazelzet JA, de Groot R, van Mierlo G, Joosten KF, van der Voort E, Eerenberg A, et al. Complement activation in relation to capillary leakage in children with septic shock and purpura. Infect Immun. 1998 Nov;66(11): 5350-6.

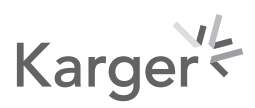


14 Berends ET, Kuipers A, Ravesloot MM, Urbanus RT, Rooijakkers SH. Bacteria under stress by complement and coagulation. FEMS Microbiol Rev. 2014 Nov;38(6):1146-71.

15 Wu F, Zou Q, Ding X, Shi D, Zhu X, Hu W, et al. Complement component C3a plays a critical role in endothelial activation and leukocyte recruitment into the brain. J Neuroinflammation. 2016 Jan;13(1):23.

16 Roxvall L, Bengtson A, Sennerby L, Heideman M. Activation of the complement cascade by trypsin. Biol Chem Hoppe Seyler. 1991 Apr;372(4):273-8.

17 Gloor B, Stahel PF, Müller CA, Schmidt OI, Büchler MW, Uhl W. Predictive value of complement activation fragments $\mathrm{C} 3 \mathrm{a}$ and sC5b-9 for development of severe disease in patients with acute pancreatitis. Scand J Gastroenterol. 2003 Oct;38(10):1078-82.

18 Bettac L, Denk S, Seufferlein T, Huber-Lang M. Complement in pancreatic disease - perpetrator or savior? Front Immunol. 2017 Jan; 8:15.

19 Merriam LT, Webster C, Joehl RJ. Complement component C5 deficiency reduces edema formation in murine ligation-induced acute pancreatitis. J Surg Res. 1997 Jan;67(1):40-5.

20 Bhatia M, Saluja AK, Singh VP, Frossard JL, Lee HS, Bhagat L, et al. Complement factor C5a exerts an anti-inflammatory effect in acute pancreatitis and associated lung injury. Am J Physiol Gastrointest Liver Physiol. 2001 May;280(5):G974-8.

21 Leffler J, Martin M, Gullstrand B, Tydén H, Lood C, Truedsson L, et al. Neutrophil extracellular traps that are not degraded in systemic lupus erythematosus activate complement exacerbating the disease. J Immunol. 2012 Apr;188(7):3522-31.

22 Wang H, Wang C, Zhao MH, Chen M. Neutrophil extracellular traps can activate alternative complement pathways. Clin Exp Immunol. 2015 Sep;181(3):518-27.

23 Laukkarinen JM, Van Acker GJ, Weiss ER, Steer ML, Perides G. A mouse model of acute biliary pancreatitis induced by retrograde pancreatic duct infusion of Na-taurocholate. Gut. 2007 Nov;56(11):1590-8.

24 Asaduzzaman M, Zhang S, Lavasani S, Wang Y, Thorlacius H. LFA-1 and MAC-1 mediate pulmonary recruitment of neutrophils and tissue damage in abdominal sepsis. Shock. 2008 Sep;30(3):254-9.

25 Swamydas M, Lionakis MS. Isolation, purification and labeling of mouse bone marrow neutrophils for functional studies and adoptive transfer experiments. J Vis Exp. 2013 Jul; 77:e50586.

26 Schmidt J, Rattner DW, Lewandrowski K, Compton CC, Mandavilli U, Knoefel WT, et al. A better model of acute pancreatitis for evaluating therapy. Ann Surg. 1992 Jan;215(1):44-56.

27 Ricklin D, Mastellos DC, Reis ES, Lambris JD. The renaissance of complement therapeutics. Nat Rev Nephrol. 2018 Jan;14(1):26-47.

28 Goldstein IM, Cala D, Radin A, Kaplan HB, Horn J, Ranson J. Evidence of complement catabolism in acute pancreatitis. Am J Med Sci. 1978 May-Jun;275(3):257-64.

29 Minta JO, Man D, Movat HZ. Kinetic studies on the fragmentation of the third component of complement (C3) by trypsin. J Immunol. 1977 Jun;118(6):2192-8.

30 Linders J, Madhi R, Rahman M, Mörgelin M, Regner S, Brenner M, et al. Extracellular cold-inducible RNAbinding protein regulates neutrophil extracellular trap formation and tissue damage in acute pancreatitis. Lab Invest. 2020 Dec;100(12):1618-30.

31 Madhi R, Rahman M, Mörgelin M, Thorlacius H. c-Abl kinase regulates neutrophil extracellular trap formation, inflammation, and tissue damage in severe acute pancreatitis. J Leukoc Biol. 2019 Aug;106(2):455-66.

32 Madhi R, Rahman M, Taha D, Mörgelin M, Thorlacius H. Targeting peptidylarginine deiminase reduces neutrophil extracellular trap formation and tissue injury in severe acute pancreatitis. J Cell Physiol. 2019 Jul; 234(7):11850-60.

33 Hartwig W, Jimenez RE, Fernandez-del Castillo C, Kelliher A, Jones R, Warshaw AL. Expression of the adhesion molecules Mac-1 and L-selectin on neutrophils in acute pancreatitis is protease- and complement-dependent. Ann Surg. 2001 Mar;233(3):371-8.

34 Hartwig W, Klafs M, Kirschfink M, Hackert T, Schneider L, Gebhard MM, et al. Interaction of complement and leukocytes in severe acute pancreatitis: potential for therapeutic intervention. Am J Physiol Gastrointest Liver Physiol. 2006 Nov;291(5):G844-50. 